\title{
Un brote epidémico de endocarditis por Pseudomonas aeruginosa secundario a angiografía coronaria
}

\author{
Derya Seyman, Dilara Inan, Nevgun. Sepin Ozen y Dilara Ogunc
}

\section{An outbreak of Pseudomonas aeruginosa infective endocarditis subsequent to coronary angiography}

Objectives: To describe an endocarditis outbreak affecting three patients due to Pseudomonas aeruginosa infection post coronary angiography performed in the Cardiovascular Surgery and Cardiology Medical Center of a private hospital. Methods: After recognition of an infection cluster within a onemonth period, the outbreak was reported to Antalya Department of Health and a broad investigation was initiated in order to determine the most probable cause and/or source of nosocomial pseudomonal endocarditis. Patient data were obtained by medical record review as well as interviews with patients or their next of kin. Thirty-six surveillance samples for $P$. aeruginosa were collected from various locations within the coronary angiography unit. The outbreak research team reviewed the private hospital's Cardiovascular Surgery and Cardiology Medical Center's infection control procedures. The epidemiology of $P$. aeruginosa was studied through analysis of phenotypic markers, including antimicrobial sensitivity profiles. Results: The infection control audit revealed multiple breaches of infection control procedures. Only 1/36 environmental samples yielded, which was isolated from a radio-opaque solution within an angiography injector pump. P. aeruginosa from the radio-opaque solution had an identical antimicrobial susceptibility pattern to the strain isolated from patients. Both samples were susceptible to all antipseudomonal agents. This outbreak could have been successfully controlled by instituting combined infection control measures. Conclusions: This outbreak emphasizes the important of adherence to infection control standards and practices for cardiac catheterization, as well as the need for closer collaboration between the Infection Control Committee and coronary angiography personnel.

Key words: Pseudomonas aeruginosa, infective endocarditis, coronary angiography, outbreak, infection control.

\section{Introducción}

$P$ seudomonas aeruginosa es un patógeno gramnegativo patógeno causante de infecciones nosocomiales y en hospederos inmunocomprometidos asociándose con alta morbi-mortalidad. Pseudomonas aeruginosa prospera en ambientes húmedos, tales como el agua, la tierra y la piel $^{1}$. Tiene mínimos requerimientos nutricionales, pese a su amplia capacidad de adaptarse a ecosistemas. La contaminación nosocomial de $P$. aeruginosa ocurre habitualmente por intermedio de los equipamientos médicos y quirúrgicos, medicamentos, desinfectantes y el jabón. En la literatura médica se ha reportado brotes de $P$. aeruginosa causados por diversos procedimientos médicos, incluyendo broncoscopio contaminado, colangio-pancreatografía retrógrada endoscópica y cistoscopia ${ }^{2-4}$. La contaminación de soluciones salinas multidosis heparinizadas ha sido demostrada como fuente ambiental de bacteriemias nosocomiales ${ }^{5}$.

La coronariografía (angiografía coronaria-AC) es un procedimiento seguro pero invasor, utilizada comúnmente para tanto diagnóstico como tratamiento de una enferme- dad coronaria ${ }^{6}$. Estudios prospectivos sobre bacteriemia post cateterización cardiaca (CC) señalan bajos índices de infección ${ }^{7,8}$. Aunque se han descrito diversos casos de endocarditis infecciosa (EI) tras efectuarse CC, no se han comunicado brotes epidémicos ${ }^{9,10}$.

Staphylococcus aureus y Staphylococcus coagulasa negativa son componentes de la microbiota cutánea del ser humano. Estas especies bacterianas invaden usualmente el torrente sanguíneo por colonizar las líneas vasculares o penetrar la piel durante su instalación. Son, por tanto, las causas más comunes de bacteriemia y $\mathrm{EI}^{11}$. Una revisión reciente de la literatura médica en endocarditis post CC describe nueve casos, en siete de los cuales se aisló una especie bacteriana grampositiva ${ }^{12}$.

La endocarditis por Pseudomonas aeruginosa es una entidad clínica inusual, frecuentemente hallada en pacientes consumidores de drogas intravenosas (CDIV) ${ }^{13}$. La EI por $P$. aeruginosa es típicamente una infección nosocomial esporádica, de muy difícil tratamiento y elevada morbilidad y letalidad ${ }^{14}$. El estudio de una cohorte multinacional, prospectiva, de EI por bacilos gramnegativos no-HACEK (Haemophilus sp, Aggregatibacter actinomycetemco-
Antalya Education and Research Hospital, Antalya, Turquía. Department of Infectious Diseases and Clinical Microbiology (DS). Akdeniz University Antalya, Turquía.

Department of Infectious Diseases and Clinical Microbiology (DI). Department of Medical Microbiology (DO). Antalya Hifzıssıhha Institute, Antalya, Turquía. Department of Clinical Microbiology (NSO).

Conflictos de interés: Todos los autores declaran no tener relación comercial o haber recibido dineros en relación al contenido de este artículo.

Recibido: 17 de enero de 2013 Aceptado: 21 de marzo de 2014

Correspondencia a: Dilara Inan inan@akdeniz.edu.tr 
mitans -antes Actinobacillus actinomycetemcomitans-, Cardiobacterium hominis, Eikenella corrodens y Kingella sp) encontró que $P$. aeruginosa fue responsable de $22 \%$ $(11 / 49)$ de todos los casos, de los cuales $57 \%$ tuvieron un origen asociado a la atención de salud ${ }^{15}$.

En este escrito, comunicamos la investigación de un brote de EI por $P$. aeruginosa que afectó a pacientes sometidos a AC. Consideramos que ésta es la primera comunicación en la literatura médica de un brote de EI causada por $P$. aeruginosa como consecuencia de haberse efectuado AC.

\section{Métodos}

\section{Detección del brote epidémico}

En el plazo de un mes se diagnosticaron tres casos de EI, según los criterios de Duke's ${ }^{16}$, causados por $P$. aeruginosa en un mismo hospital docente en Antalya, Turquía; se trataba de un agente etiológico insospechado como causa de EI en válvula nativa o protésica ${ }^{13,17}$. Por ende, sospechamos que se trataba de un brote epidémico y se inició de inmediato una investigación epidemiológica para establecer si la AC era un factor de riesgo para adquirir la EI por $P$. aeruginosa o no. En primer lugar, se revisaron los registros clínicos de los tres pacientes y sus historiales médicos a la busca de posibles factores de riesgo de transmisión de $P$. aeruginosa. Cuando fue factible, se efectuaron entrevistas presenciales a los pacientes, en caso contrario se obtuvo información de sus parientes. Esta evaluación incluyó factores demográficos, el momento y curso de los síntomas en relación con la $\mathrm{AC}$, síntomas presentados, complicaciones y resolución de la enfermedad, registros ecocardiográficos y factores de riesgo para transmisión de $P$. aeruginosa, tales como diabetes mellitus, tuberculosis, SIDA, cáncer, terapia corticoesteroidal u otra terapia inmunosupresora, CDIV, asistencia a spa y piscina, hospitalizaciones previas (definidas como hospitalización dentro de los 30 días anteriores a la hospitalización actual), procedimientos invasores recientes (definidos como intervenciones en los últimos tres meses antes de la hospitalización actual) o historia de instalación de catéter urinario, vascular o cualquier otro dispositivo invasor.

El único factor de riesgo común a los tres pacientes fue que todos habían sido sometidos a una $\mathrm{AC}$, en un mismo centro médico de cardiología y cirugía cardiovascular (CMC-CCV) privado, en el área metropolitana de Antalya, dentro del 1,5 mes reciente.

\section{Organización de un team de investigación epidemiológica}

Una vez establecida la relación entre la AC y las EI causadas por P. aeruginosa, el Departamento de Salud de Antalya (DS-A) fue notificado inmediatamente mediante un documento oficial que incluía una descripción de nuestra hipótesis que el brote de endocarditis era debido a $P$. aeruginosa, la fuente de origen más posible, y la presentación clínica de los tres casos. El DS-A invitó a tres hospitales estatales vecinos a constituir un team de investigación del brote (TIB), el que fue conformado por tres infectólogos, dos enfermeras de control de infecciones y un cardiólogo. El TIB visitó este CMC-CCV privado con el objeto de investigar la fuente de la transmisión de $P$. aeruginosa.

Con la finalidad de detector más casos, todas las instituciones de salud y hospitales en Antalya fueron contactados y consultados si tenía algún caso reciente o simultáneo de becteriemia o EI por $P$. aeruginosa originado en la comunidad, en especial en pacientes que hubieran sido sometidos recientemente a una AC.

\section{Vigilancia epidemiológica e intervenciones de control de infecciones}

Obtención de muestras medioambientales. Se tomaron muestras ambientales con tórulas humedecidas con solución salina fisiológica estéril. Además, se obtuvieron muestras de líquidos mediante aspiración con jeringas. Todas estas muestras fueron enviadas rápidamente en medios de transporte al Laboratorio de Microbiología de la Akdeniz University. Las tórulas húmedas y los líquidos fueron sembrados directamente en agar sangre de cordero al $5 \%$ y placas de agar eosina-azul de metileno. Se incubó las placas en aerobiosis a $35^{\circ} \mathrm{C}$ por $24-48 \mathrm{~h}$. La identificación de las cepas y el estudio de susceptibilidad in vitro fueron efectuados con el sistema automatizado Phoenix (Phoenix; Diagnostic Instrument Systems; Becton Dickinson, USA).

Auditoria de las intervenciones de control de infecciones. El TIB y tres funcionarios del DS-A efectuaron una visita inspectiva, sin previo aviso, al CMC-CCV privado. El TIB evaluó la implementación de la National Infection Control and Prevention Regulation, que contempla la habilitación de instalaciones para la adecuada higiene de manos, equipos de protección personal, servicios de ropería y lavandería, descarte de agujas y otros objetos corto-punzantes, manejo de desechos clínicos, procesamiento de instrumentos y equipos re-utilizables, manejo de la higiene ambiental y derrames, e implementación de requerimientos para educación y entrenamiento. El team revisó también el programa y las políticas de control de infecciones del establecimiento. El TIB vigiló también, en forma directa, si el procedimiento de AC era efectuado en forma correcta, lo que incluyó: 1) preparación y protección del paciente; 2) técnica de cateterización cardiaca; 3) higiene de manos; 4) precauciones máximas de barreras estériles, incluyendo el uso de gorro, mascarilla, protección ocular, delantal y guantes estériles; 5) manejo de dispositivos desechables, medicaciones, medios de contraste y soluciones a infundir; y 6) mantenimiento del 
medioambiente del pabellón de cateterización cardiaca, incluyendo una limpieza apropiada, ventilación y restricción de la circulación del personal.

\section{Resultados}

\section{Paciente 1}

Un adulto mayor, de 64 años ingresó a nuestro hospital con historia de fiebre recurrente, pérdida de peso y cansancio en aumento. Su historial médico incluía una hipertensión arterial. Negó tener factores de riesgo relativos a EI, incluyendo soplo cardiaco, valvulopatía cardiaca y recientes intervenciones dentales. Sólo un mes antes de esta hospitalización se le había efectuado una AC, en forma ambulatoria, por una angina inestable. Relataba una fiebre de origen desconocido.

Hallazgos relevantes al examen físico fueron: una temperatura de $38,8^{\circ} \mathrm{C}$ y un soplo de eyección pansistólico en el ápex. Se le practicó de inmediato una ecocardiografía trans-torácica (ETT), detectándose una vegetación de 12 × $9 \mathrm{~mm}$ adherida al velo anterior de la válvula mitral, y un velo posterior protruyendo hacia la aurícula izquierda. El laboratorio indicaba: recuento de leucocitos $15.800 / \mathrm{mm}^{3}$, hemoglobina $11,7 \mathrm{~g} / \mathrm{dL}$, PCR $15,55 \mathrm{mg} / \mathrm{dL}$, y los exámenes restantes, incluyendo medición de $\mathrm{C} 3$ y $\mathrm{C} 4$, estaban en valores normales. El análisis de orina reveló eritrocitos 10-15 y leucocitos 8-10 por campo, con ausencia de bacterias. Una ultrasonografía abdominal fue normal. Se obtuvieron cultivos de sangre y orina al momento de ingresar el paciente y se inició en forma empírica tratamiento con $8 \mathrm{~g} /$ día de ampicilina/sulbactam, $4 \mathrm{~g} /$ día de ampicilina, y $1 \mathrm{mg} / \mathrm{kg}$ de gentamicina cada $8 \mathrm{~h}$ con el diagnóstico de EI. Los cuatro hemocultivos fueron positivos para $P$. aeruginosa sensible a todos los agentes anti-pseudomonas.

Se ajustó entonces la terapia antimicrobiana a $3 \mathrm{~g} /$ día de ceftazidima y $1 \mathrm{~g} /$ día de amikacina. La fiebre se resolvió completamente al día 4 de este segundo tratamiento. La PCR se normalizó durante la tercera semana de antibioterapia Los hemocultivos de control fueron negativos. La ETT se repitió en forma semanal durante la hospitalización y evidenció una reducción paulatina de su tamaño. Terminado este tratamiento el paciente no presentaba falla cardiaca, de tal manera que no requirió reemplazo valvular.

Esta terapia asociada fue mantenida durante seis semanas. El paciente se veía muy estable clínicamente y fue dado de alta sin continuar antibioterapia. Una ETT demostró cicatrización de la vegetación dos meses más tarde. Su seguimiento no detectó recaída de la EI.

\section{Paciente 2}

Un adulto mayor de 61 años, con historia de hipertensión arterial había sido ingresado al Departamento de Neurología tres semanas antes con el diagnóstico de Ataque Isquémico Transitorio (AIT). Sin embargo, ya en casa, comenzó a presentar fiebre y, una semana tras su egreso fue nuevamente hospitalizado por un segundo AIT. En esta ocasión y dado su curso febril, el paciente fue evaluado por el Departamento de Infectología. No tenía factores comunes de riesgo para desarrollar una EI, pero aproximadamente 1,5 meses antes, había sido sometido a una $\mathrm{AC}$ a raíz de un dolor torácico de causa no precisada.

Hallazgos destacables en su examen físico eran: fiebre de $39,7^{\circ} \mathrm{C}$ acompañada de calofríos solemnes, pérdida de la motricidad en grado $3 / 5$ de las extremidades superior e inferior derechas, borde hepático palpable $3 \mathrm{~cm}$ bajo el reborde costal derecho, y un soplo sistólico de eyección, $3 / 6$, en el borde esternal izquierdo. No había estigmas periféricos de EI.

Los análisis de laboratorio mostraban: recuento leucocitario de $19.870 / \mathrm{mm}^{3}$, hemoglobina de $10,9 \mathrm{~g} / \mathrm{dL}$, PCR de $31 \mathrm{mg} / \mathrm{dL}$, creatininemia $1,3 \mathrm{mg} / \mathrm{dL}$. El resto de los exámenes químicos eran normales. Habiendo obtenido hemocultivos, se inició una antibioterapia combinada de cefepime y amikacina, considerando el antecedente de la hospitalización en el mes previo. Una ETT demostró la existencia de una vegetación libremente móvil de 12 x 6 $\mathrm{mm}$ adherida a la válvula mitral, junto a cierto grado de insuficiencia mitral. A los tres días de incubación, se aisló $P$. aeruginosa en todos los hemocultivos. Se constató que esta cepa tenía el mismo antibiograma que el paciente 1. La antibioterapia en curso se mantuvo y, en un control ecocadiográfico, se apreció una reducción del tamaño de la vegetación. No obstante, en el día 24 de antibioterapia, el paciente presentó signos de una falla cardiaca aguda. Fue trasladado a la unidad de cuidados intensivos (UCI) y apoyado con ventilación mecánica. Se le sometió a cirugía cardiaca con reemplazo mitral por una válvula protésica. Diez días tras ser operado, presentó un tamponamiento cardiaco, drenándose quirúrgicamente abundante fluido. La condición clínica del paciente mejoró, pero 16 días después de esta cirugía, reapareció la fiebre, acompañada de leucocitosis, secreción respiratoria purulenta, y una efusión pleural masiva. Se cambió la terapia a meropenem y se le efectuó una toracostomia a izquierda. Se comprobó la existencia de una neumonía asociada a la ventilación mecánica, por Acinetobacter baumannii.: La condición clínica y hemodinámica del paciente empeoró y finalmente falleció.

\section{Paciente 3}

Una mujer de 28 años de edad, con malestar general, fiebre, dificultad respiratoria y deterioro en su estado general fue ingresada en nuestra UCI de cirugía cardiovascular. $\mathrm{Su}$ historial médico anotaba el antecedente de fiebre y cardiopatía reumática, causantes de una grave disfunción mitro-aórtica. Un mes antes se le había sometido a una AC 
y reemplazo aórtico y mitral. No presentó incidentes en el perioperatorio ni post-operatorio. Negaba haber recibido procedimientos dentales, diagnósticos o terapéuticos, que constituyeran riesgo para desarrollar una EI.

En su evaluación clínica inicial, su temperatura era de $38,6^{\circ} \mathrm{C}$, pulso 112 latidos/min, frecuencia respiratoria $28 /$ min, y presión arterial 100/50 $\mathrm{mmHg}$. La saturación de oxígeno era $78 \%$ respirando aire ambiental. $\mathrm{Su}$ estado mental era confuso. La evaluación cardiovascular reveló ingurgitación de las venas yugulares, los ruidos protésicos y soplos aórtico y mitral en intensidad de 3/6. El examen del aparato respiratorio evidenció disminución del murmullo respiratorio en ambas bases y campos medios pulmonares. Había además, edema en las extremidades inferiores.

Los principales hallazgos de laboratorio eran: hemoglobina $8,9 \mathrm{~g} / \mathrm{dL}$, recuento de leucocitos $39,200 / \mathrm{mm}^{3}$, PCR $42 \mathrm{mg} / \mathrm{dL}$, creatininemia $5 \mathrm{mg} / \mathrm{dL}$. Un ETT de emergencia comprobó la dehiscencia de la válvula aórtica. Se planteó un shock séptico complicado con una EI iniciándose tratamiento con inótropos y antbioterapia empírica con meropenem (500 mg cada $24 \mathrm{~h}$ ) and vancomicina (1 g cada 4 días). Dos hemocultivos detectaron la presencia de $P$. aeruginosa; entonces se suspendió vancomicina y se adicionó amikacina. Apenas la condición hemodinámica del paciente lo permitió, se efectuó un reemplazo valvular aórtico, el día 5 desde su ingreso. Los hemocultivos obtenidos tras la operación fueron negativos. El examen patológico de la válvula protésica dio hallazgos compatibles con una EI. El cultivo de esta pieza fue negativo. Al día 9 post-operatorio, los gases arteriales se deterioraron en forma brusca y el paciente falleció en pocas horas, pese a las intervenciones médicas efectuadas.

\section{Revisión del control de infecciones}

El TIB investigó los registros médicos del programa de control de infecciones relativas a las AC. Esta auditoría constató la ausencia de un programas o políticas escritas para el control de infecciones, la inexistencia de un Comité de Control de Infecciones conformado por enfermeras y médicos calificados en el tema, como tampoco la existencia de protocolos para los procedimientos de esterilización y desinfección. Esto, contraviniendo la normativa que en Turquía, todos los hospitales públicos como privados debieran elaborar e implementar activamente un programa de control de infecciones.

El TIB revisó también las AC y detectó que los catéteres de un uso eran descartados después de ser usados una vez pero, que la bomba desechable de inyección del medio de contraste durante la $\mathrm{AC}$ no era cambiada tras cada procedimiento en forma regular. Las guías para el control de infecciones secundarias a cateterismo cardiaco recomiendan el uso por una sola vez de los catéteres desechables o limitan la re-utilización de equipos, e incluyen el cambio de todos los receptáculos para los medios de contraste y soluciones empleadas para flush en cada AC.

Finalmente, el TIB recolectó 36 muestras de vigilancia durante la inspección a la sala de AC. Diez y siete de ellas fueron extraídas de varios líquidos de soluciones yodadas, jabón líquido, desinfectantes con base alcohólicas y soluciones de heparina; tres de agua destilada en botellas de humidificador de $\mathrm{O}_{2}$; cinco de las soluciones radioopacas y una de solución alcohólica. Las 19 muestras restantes fueron obtenidas de diversas superficies. Una sola muestra ambiental, la solución de medio radio-opaco obtenida de la bomba inyectora de la angiografía, arrojó el resultado positivo de $P$. aeruginosa. Todas la demás muestras medioambientales fueron negativas. El aislado procedente de los pacientes tenía el mismo fenotipo (perfil de susceptibilidad in vitro) que $P$. aeruginosa recuperada de la solución del medio radio-opaco. Estos aislados eran sensibles a todos los anti-pseudomónicos (ceftazidima, piperacilina, amikacina, tobramicina, piperacilina/tazobactam, gentamicina, ciprofloxacina, cefepime, imipenem y meropenem).

\section{Seguimiento de la vigilancia y resolución}

La unidad de AC de esta institución particular fue clausurada por el DS-A debido a las deficiencias regulatorias descubiertas por el TIB. El DS-A declaró que el centro médico, de acuerdo a la National Infection Control and Prevention Regulation, debiera: instituir actividades para el control de infecciones y manejo de riesgos en el control de infecciones tan pronto como fuera posible; proceder a una limpieza medioambiental, desinfección y esterilización del instrumental; instaurar una enfermera y un médico encargados del control de infecciones; entrenar al personal, y establecer por escrito políticas para el control de microorganismos nosocomiales.

Se estableció un programa de control de infecciones activamente en este centro médico en el plazo de un mes. El DS-A revisó el programa de control de infecciones y recolectó las mismas muestras medioambientales como lo hubo efectuado con anterioridad. El mismo laboratorio de microbiología evaluó todas las muestras medioambientales encontrando que todas fueron estériles. La unidad de $\mathrm{AC}$ fue autorizada entonces para reanudar normalmente sus actividades.

No se detectaron nuevos casos, incluso pese a que la información oficial acerca de este brote fuera comunicada a los hospitales vecinos. Se considera, por esta razón, que el brote relatado se limitó a los tres pacientes descritos.

\section{Discusión}

Este manuscrito describe un brote nosocomial de endocarditis causada por $P$. aeruginosa, el que comprometió a tres pacientes ambulatorios que consultaron en un mismo 
CMC-CCV. Todos ingresaron con posterioridad a nuestro hospital, en el plazo de un mes, apuntando a que se trataba de un brote. A nuestro entender, se han comunicado más de 200 casos de EI causada por $P$. aeruginos $a^{13}$. Pero no había sido publicada hasta ahora evidencia alguna de adquirir la EI por este agente como parte de un brote nosocomial.

Bicanic y cols. ${ }^{18}$, efectuaron una revisión de la literatura médica acerca de EI por P. aeruginosa sobre válvula nativa en no CDIV mediante una búsqueda en Medline desde 1966 al 2001 y encontró 17 casos. También nosotros efectuamos una búsqueda bibliográfica incluyendo todos los artículos publicados en inglés desde el 2000 al 2011 y encontramos 10 casos que cumplían los mismos requisitos $^{14,18-24}$. Ninguno de estos 27 pacientes tenía como foco primario una infección urinaria, una fístula arterio-venosa con infección del injerto, o factores predisponentes como: quemaduras, cirrosis hepática, hemodiálisis, diabetes mellitus, o procedimientos médicos o quirúrgicos recientes para desarrollar una EI por P. aeruginosa.

Ninguno de nuestros pacientes tenía factores de riesgo para adquirir una infección por $P$. aeruginosa, y el único rasgo en común en su historial médico era el haberse realizado una $\mathrm{AC}$ en el mismo centro médico. En consecuencia, la AC podría ser la fuente de la EI por $P$. aeruginosa, o al menos un factor de riesgo. Efectuado un completo estudio, la fuente del brote fue encontrada en la solución del medio radio-opaco contenida en la bomba inyectora empleada en la AC.

Al presente, sólo dos artículos han sido publicados sobre EI por $P$. aeruginosa tras cateterización cardíaca ${ }^{12,18}$. El curso clínico en estos dos artículos fue esporádico, incluyeron seis pacientes, en quienes la puerta de entrada para la infección por $P$. aeruginosa fue la piel en cinco casos y un stent infectado, en un caso. En nuestra comunicación, hay dos diferencias importantes: 1) nuestros pacientes fueron parte de un brote epidémico, y 2) la fuente de infección fue una bomba de inyección para angiografía, no la microbiota cutánea. La misma cepa de $P$. aeruginosa fue identificada fenotípicamente desde los pacientes y la bomba inyectora de angiografía; este hallazgo confirmó que la contaminación bacteriana fue adquirida durante los procedimientos de angiografía.

Fuimos capaces de dilucidar cómo se produjo la transmisión durante al AC. Descubrimos que terminado cada procedimiento, el staff no cambiaba la bomba inyectora desechable. Guías para el control de infecciones en laboratorios de cateterización cardiaca están disponibles en la literatura científica y en ellas se recomienda que todos los contenedores de medios de contraste debieran utilizarse sólo una vez y cambiados para el siguiente paciente ${ }^{25}$. De acuerdo al manejo de control de infecciones, la reutilización de envases individuales y/o contenedores de medios de contraste es el factor de riesgo más común para la contaminación durante el procedimiento de cateterización cardiaca. El centro médico alegó que ellos desconocían las guías para el control de infecciones asociadas a las CC. En consecuencia, los médicos clínicos que efectuaren angiografías debieran ser advertidos de los factores de riesgo potencial, así como la posibilidad que un paciente desarrolle complicaciones potencialmente graves incluyendo una EI, después de una AC. También debieran los médicos tratantes estar muy de acuerdo con los Comités de Control de Infecciones acerca de las guías de protocolos para la desinfección y control de infecciones.

Normalmente, en los sistemas de vigilancia, las infecciones por $P$. aeruginosa no dejan de ser reportadas; no obstante, no todos los procedimientos invasores tienen el seguimiento necesario para detectar eventos adversos posteriores como son las infecciones. Los brotes de infecciones debidos a equipos contaminados ocurren por errores cometidos en su desinfección ${ }^{4}$. Los programas de vigilancia activa basada en la unidad de AC parecen ser la manera más efectiva de minimizar los riesgos de EI consecutivos a una AC.

No encontramos comunicaciones previas sobre la contaminación de medios radio-opacos como causa de EI. Creemos que esta es la primera comunicación al respecto. Esta investigación tiene, no obstante, ciertas limitaciones. En primer lugar, no fue efectuada una tipificación molecular porque las cepas de $P$. aeruginosa susceptibles in vitro no son almacenadas comúnmente por el laboratorio de Microbiología de la Akdeniz University. Tal fue así, que las cepas aisladas en el brote fueron eliminadas antes de haberse detectado este brote epidémico.

En segundo lugar, no hicimos un estudio caso-control, debido a que es imposible conocer el número de pacientes ambulatorios a quienes se les efectuó una $\mathrm{AC}$ ya que los registros médicos no son almacenados en este centro. En este CMC-CCV privado, se realizan diariamente entre 8 y $12 \mathrm{AC}$ en promedio, lo que se traduce en un elevado número de procedimientos de $\mathrm{AC}$ al mes

Con el propósito de encontrar la fuente de infección en un brote epidémico, es necesario un esfuerzo cooperativo una vez que el brote ha sido detectado. Durante esta investigación, el DS-A trabajó en coordinación con el TIB conformado. La fuente de infección fue identificada debido a una esfuerzo extra del TIB, el que incluso ofreció con tomar medidas necesarias para evitar la diseminación del brote. El DS-A se encargó de vigilar que se cumplieran las recomendaciones del TIB; un mes más tarde, los cultivos de control fueron estériles. El brote fue controlado en forma exitosa introduciendo el uso desechable de medios de contraste en cada AC, limpieza medioambiental, esterilización apropiada y desinfección de los equipos.

En conclusión, la AC debiera asumirse como un factor de riesgo para adquirir una EI, especialmente si las medidas de control de infecciones no son observadas correctamente. La adecuada implementación de desin- 
fección y procedimientos de esterilización debieran ser controlados con acuciosidad por el Comité de Control de Infecciones para prevenir la emergencia de tales brotes. Este brote presentado re-enfatiza la importancia de las medidas para el control de infecciones.

Agradecimientos. Los autores agradecen al Antalya Department of Health por la coordinación de estos estudios.

\section{Resumen}

Objetivos: Describir un brote de endocarditis por Pseudomonas aeruginosa que afectó a tres pacientes tras habérseles efectuado una coronariografía en el Centro Médico de Cardiología y de Cirugía Cardiovascular (CMC-CCV) de un hospital privado. Métodos: Después de reconocer la aparición de un brote en un periodo de un mes, este hecho fue comunicado al Departamento de Salud de Antalya, iniciándose una exhaustiva investigación para precisar la más probable causa y/o fuente de las endocarditis nosocomiales. Se extrajo de los registros médicos los datos clínicos de los pacientes y se efectuaron entrevistas a los pacientes o sus familiares. Se extrajo 36 muestras medioambientales de vigilancia en busca de $P$. aeruginosa de diversos sitios dentro de la unidad de coronariografía.
Un team que investigó el brote revisó los procedimientos en uso para la prevención de infecciones en el CMCCCV. Se estudió la epidemiología de la $P$. aeruginosa mediante análisis de su fenotipos, incluyendo el perfil de susceptibilidad in vitro a antimicrobianos. Resultados: La auditoria comprobó el quiebre de diversas normas de control de infecciones. Sólo $1 / 36$ de las muestras ambientales arrojó el cultivo de $P$. aeruginosa, a partir de una solución de medio radio-opaco dentro de una bomba inyectora empleada en las angiografías. Los aislados de P. aeruginosa desde la solución del medio radio-opaco tenían idéntico patrón de susceptibilidad antimicrobiana que las cepas recuperadas de los pacientes. Ambos tipos de muestras eran susceptibles a todos los antimicrobianos con actividad anti-pseudomonas. El brote pudo evitarse si se hubieran instaurado una serie de medidas de control de infecciones. Conclusiones: Este brote enfatiza la importancia de adherir a los estándares y prácticas de control de infecciones para la cateterización cardiaca, así como la necesidad de una estrecha colaboración entre el Comité de Control de Infecciones y el personal involucrado en el procedimiento de coronariografía.

Palabras clave: Pseudomonas aeruginosa, endocarditis infecciosa, angiografía coronaria, brote epidémico, control de infecciones.

\section{Referencias bibliográficas}

1.- Trautmann M, Lepper P M, Haller M. Ecology of Pseudomonas aeruginosa in the intensive care unit and the evolving role of water outlets as a reservoir of the organism. Am J Infect Control 2005; 33: 41-9.

2.- Corne $\mathrm{P}$, Godreuil S, Jean-Pierre H, Jonquet $\mathrm{O}$, Campos J, Jumas-Bilak E, et al. Unusual implication of biopsy forceps in outbreaks of Pseudomonas aeruginosa infections and pseudo-infections related to bronchoscopy. J Hosp Infect 2005; 61: 20-6.

3.- Fraser T G, Reiner S, Malczynski M, Yarnold P R, Warren J, Noskin G A. Multidrug-resistant Pseudomonas aeruginosa cholangitis after endoscopic retrograde cholangiopancreatography: failure of routine endoscope cultures to prevent an outbreak. Infect Control Hosp Epidemiol 2004; 25: 856-9.

4.- Wendelboe A M, Baumbach J, Blossom D B, Frank P, Srinivasan A, Sewell C M. Outbreak of cystoscopy related infections with Pseudomonas aeruginosa: New Mexico, 2007. J Urol 2008; 180: 588-92.

5.- Prospero E, Barbadoro P, Savini S, Manso E, Annino I, D'Errico M M. Cluster of Pseudomonas aeruginosa catheter-related bloodstream infections traced to contaminated multidose heparinized saline solutions in a medical ward. Int J Hyg Environ Health 2006; 209: 553-6.

6.- Matthews P C, Missouris C G, Jordaan J, Lessing M P. Staphylococcus lugdunensis endocarditis following cardiac catheterisation. Int J Cardiol 2008; 130: 87-8.

7.- Banai S, Selitser V, Keren A, Benhorin J, Shitrit O B, Yalon S, et al. Prospective study of bacteremia after cardiac catheterization. Am J Cardiol. 2003; 92: 1004-7.

8.- Shea K W, Schwartz R K, Gambino A T, Marzo K P, Cunha B A. Bacteremia associated with percutaneous transluminal coronary angioplasty. Cathet Cardiovasc Diagn 1995; 36: 5-9.

9.- Benito Bartolomé F, Sánchez FernándezBernal C. Infectious mitral endocarditis after radiofrequency catheter ablation of a left lateral accessory pathway. Rev Esp Cardiol 2001; 54: 999-1001.

10.- McCready R A, Siderys H, Pittman J N, Herod G T, Halbrook H G, Fehrenbacher J W, et al. Septic complications after cardiac catheterization and percutaneous transluminal coronary angioplasty. J Vasc Surg 1991; 14: 170-4.

11.- Samore M H, Wessolossky M A, Lewis S M, Shubrooks S J Jr, Karchmer A W. Frequency, risk factors, and outcome for bacteremia after percutaneous transluminal coronary angioplasty. Am J Cardiol 1997; 79: 873-7.
12.- Jayasuriya S, Movahed M R. Infectious endocarditis with systemic septic embolization as a rare complication of cardiac catheterization. Exp Clin Cardiol 2009; 14: 17-20.

13.- Fowler V G, Scheld W M, Bayer A S. Endocarditis and intravascular infections. In: Mandell, Douglas \& Bennett's Principles and Practice of Infectious Diseases. Mandell GL, Bennett JE, Dolin R, editors. 6th ed. Churchill Livingstone, Philadelphia, 2005: 975-1022.

14.- Durante-Mangoni E, Tripodi M F, Albisinni R, Utili R. Management of Gram-negative and fungal endocarditis.Int J Antimicrob Agents. 2010; 36: 40-5.

15.- Morpeth S, Murdoch D, Cabell C H, Karchmer A W, Pappas P, Levine D, et al. Non-HACEK gram-negative bacillus endocarditis. Ann Intern Med. 2007; 147: 829-35.

16.- Li J S, Sexton D J, Mick N, Nettles R, Fowler V G Jr, Ryan T et al. Proposed modifications to the Duke criteria for the diagnosis of infective endocarditis. Clin Infect Dis 2000; 30: 633-8.

17.- Erbay A R, Erbay A, Canga A, Keskin G, Sen N, Atak R, et al. Risk factors for in-hospital mortality in infective endocarditis: five years experience at a tertiary care hospital in Turkey. J Heart Valve Dis 2010; 19: 216-24.

18.- Bicanic T A, Eykyn S J. Hospital-acquired, native valve endocarditis caused by 
Pseudomonas aeruginosa. J Infect 2002; 44: 137-9.

19.- Venkatesan A, Spalding C, Speedie A, Sinha G, Rumbaugh JA. Pseudomonas aeruginosa infective endocarditis presenting as bacterial meningitis. J Infect 2005; 51: 199-202.

20.- Sá MI, Môço R, Cabral S, Reis A H, Pereira L S, Torres S, et al. Isolated pulmonary valve endocarditis due to Pseudomonas aeruginosa. Rev Port Cardiol 2007; 26: 43-8.

21.- Ordóñez K M, Hernández O A, Cortés J A,
López M J, Alfonso G, Junca A. Left-sided infective endocarditis caused by Pseudomonas aeruginosa treated medically. Biomedica. 2010; 30: 164-9.

22.- Hsu R B, Chen R J, Chu S H. Infective endocarditis in patients with liver cirrhosis. J Formos Med Assoc 2004; 103: 355-8

23.- Gouëllo J P, Asfar P, Brenet O, Kouatchet A, Berthelot G, Alquier P. Nosocomial endocarditis in the intensive care unit: an analysis of 22 cases. Crit Care Med 2000; 28: 377-82.
24.- Hsu K H, Ben R J, Shiang J C, Feng N H. Pseudomonas aeruginosa endocarditis associated with endophthalmitis caused by arteriovenous fistula and graft infection. J Chin Med Assoc 2003; 66: 617-20.

25.- Chambers C E, Eisenhauer M D, McNicol L B, Bloc P C, Phillips W J, Dehmer G J, et al. Infection control guidelines for the cardiac catheterization laboratory: society guidelines revisited. Catheter Cardiovasc Interv 2006; 67: 78-86. 